\title{
An Efficient Power Aware Algorithm for Optimizing Energy Consumption of Cloud Resources Using Multi Agent Model
}

\author{
Prabha.B ${ }^{1}$, Ramesh. $\mathrm{K}^{2}$, Renjith $\mathrm{P} \mathrm{N}^{3}$, Aiswarya. $\mathrm{S}^{4}$ \\ \{prabha.bala32@gmail.com ${ }^{1}$, \\ rameshk.me@gmail.com ${ }^{2}$,pnrenjith@hindustanuniv.ac.in ${ }^{3}$,aiswaryas90@gmail.com ${ }^{4}$ \} \\ Research Scholar/CSE, Hindustan Institute of Technology and Science, Padur and Assistant \\ Professor/IT, Loyola-ICAM College of Engineering and Technology, Chennai ${ }^{1}$,Professor/CSE, \\ Hindustan Institute of Technology and Science,Padur ${ }^{2}$, Assoc Professor/CSE, Hindustan Institute of \\ Technology and Science, Padur ${ }^{3}$, Research Scholar/CSE, Hindustan Institute of Technology and Science, \\ Padur $^{4}$
}

\begin{abstract}
Cloud computing offers different kinds of resources to the user based on the current need. The resource includes hardware, software and platform through virtualization. User requirements are mapped to the corresponding Virtual Machine (VM) by the host of the data center. Every resource in the data center consumed a considerable amount of energy. The existing power aware models are used to minimize the energy consumption but it follows only one specific layer in the cloud. These methods suffer from performance problems due to excess energy by the idle resources. The resources like data center, host and VM consumed energy in case of idle workload and minimum workload. Idle resources are identified and removed from the resource list in order to minimize the excess energy consumption. The resource with the minimum workload is migrated to the other suitable resources using the migration method. The proposed power aware model uses multi agent support for minimizing the energy in Data center, Host and VM level. The overall analysis is carried out based on the performance parameter like Service Level Agreement (SLA) based host shutdown and violation etc. The proposed method consumes minimum energy when compared to other existing models. It achieves maximum performance by considering power aware parameters in the cloud.
\end{abstract}

Keywords: Cloud Computing, Hypervisor, Cloud Power Energy Model,Virtual Machine, Data Center, Agent Model.

\section{Introduction}

Cloud computing consists of various services which are offered to the user by using virtualization. Virtualization process is used to map the physical machine to a virtual machine for handling the user task. An infrastructure resource consumes electricity that produces the emissions, so it is minimized in proper level for making the cloud in energy efficient computing. Efficient model related to energy is handled by consolidating the cloud based on the utilization level. It also includes the hardware, functional level and nodes such as VM, physical host etc. Green computing applied over the cloud for saving the energy based on the factors such as design and production of the digital element in the respective environment. It 
also minimizes the operational as well as energy consumed by the cloud components. The energy utilization of the cloud is optimized using various techniques such as dynamic voltage method, VM method, Migration level and Consolidation of VM level and scaling of frequency level. Cloud runs a huge number of hosts with supporting elements in all times with highenergy consumption. Various algorithms are applied to reduce the energy with the consideration of the performance parameters related to the environment. Multiple algorithms are integrated for handling better energy efficient models. The policies can be created based on energy optimization by terminating the physical host and networking elements with reduced cost model [1]. The cloud resources are scattered among various regions with highend computational elements leading to the high operation cost due to the energy consumption. The energy driven approach is used over the cloud by mapping the resources to other suitable resources with less power. Cloud users are dispatching the policies with various categories such as random based, delay with cost based, delay with load based and custom policy based [2]. The best method of saving the cloud energy is to consider the VM consolidation approach in a dynamic manner. Static methods never reduce the energy because that is still running after completing the execution of the task. The manager is deployed over the consolidation process, which decides the less power resource [3]. Most of the power consumed by the cloud is CPU because of the processing capability. Recent cloud computing focuses on different service models so most of the time the CPU gets idle state. The idle CPU also consumes energy that is handled by stopping those CPUs until the request is arrived. The dynamic idle analysis and prediction is carried out for estimating the forthcoming CPU idle interval range. CPU idle power is reduced by analyzing various models and sleeping states of the CPU during runtime [4]. The energy efficiency is assessed by considering the hardware and various scheduling techniques with genetic algorithm based analysis. Bee colony algorithms are used for scheduling the task to optimize energy resources with minimum response time [5]. The renewable energy based sources are applied to the cloud data center for minimizing the cost. The fuzzy based algorithm with load balancing support is used to maximize the speed of the processor and throughput. It provides the model selection criteria in which the user selects their own cost saving model [6]. Model cloud computing uses various mobile devices that are consumed due to RF signals and other power components. The constraint is applied over the energy model with computation capability [7]. Normally the mobile clouds are performed computational oriented tasks online as well as offline. It follows the multiple flow of control from user level to remote cloud for processing of tasks [8]. Energy efficient methods of the cloud provide various advantages such as reduction in operation cost; enhance the reliability and protection of the environment and so on. Real time energy management is very difficult because of the problem in scheduling tasks to the cloud. This problem is solved by using the horizon algorithm with autonomous task [9]. The energy efficiency of the cloud is consolidated related to VM is analyzed using an energy aware framework. This model collects the utilization of all active hosts and active migrations of hosts based on the policies and load status [10]. The proposed model compares and eliminates the issues faced by the existing model by implementing the multi agent-based power aware technique with high efficiency.

\section{Related Works}

Multi agent method uses three agents at the cloud infrastructure level namely DC level, VM level and Host level. The cloud broker gets the user task and maps to the respective VM 
on the cloud for execution. The energy of all resources is assessed based on various properties then fix the reward value and terminate the useless resources which consume high energy. Fig.1. shows the agent interaction with the cloud environment. Energy aware agents are formulated from DC to VM level because the actual tasks are run on the VM. The energy is based on various factors in cloud computing so it will be handled properly in order to achieve an efficient saving of energy in cloud. Fig.2. gives the energy aware model. Single agent based algorithms are restricted to the single cloud provider with a huge number of constraints for selecting the performance parameters. This model does not save the minimum energy because of the restriction, so a multi agent method is introduced by saving more energy in all levels of the cloud environment. The proposed algorithm implements more than one agent for considering less energy resources and removes the high energy resources with limited load and idle load.

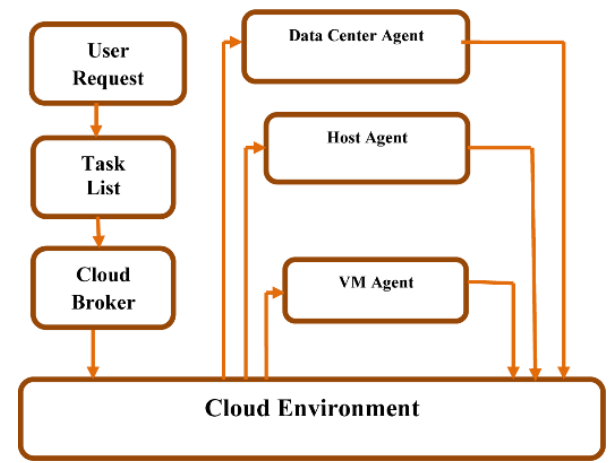

Fig. 1.Multi Agent Interaction

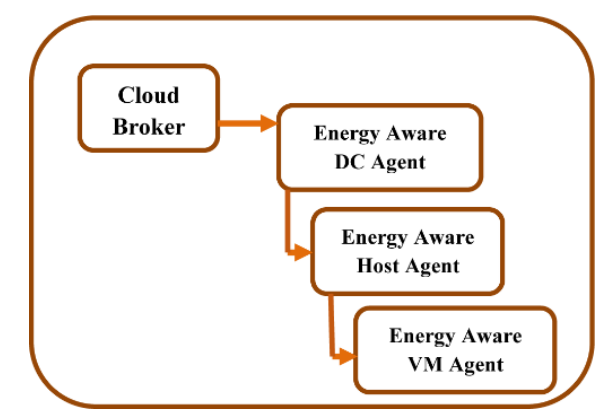

Fig. 2.Energy aware Level

\section{Proposed Energy Aware Model}

Multi agent method uses three agents at the cloud infrastructure level namely DC level, VM level and Host levThe power management of the cloud provides support to minimize the cost to the cloud user because unused resources lead to excess cost. The proposed method focuses on multi agent-based approach in which the resources are maintained based on the cloud request. The idle resources are terminated in order to reduce the 
energy consumed by the resources. Traditional agent methods use only one agent for interacting with the environment but some real time problem needs more than one environment, so it will be addressed by using more than one agent. The proposed algorithm uses a multi agent-based approach and is considered a cloud environment. Fig.3. shows that the proposed model of the multi agent algorithm. Four modules are considered for implementation namely user module, configuration module, multi agent module and cloud multi agent module.
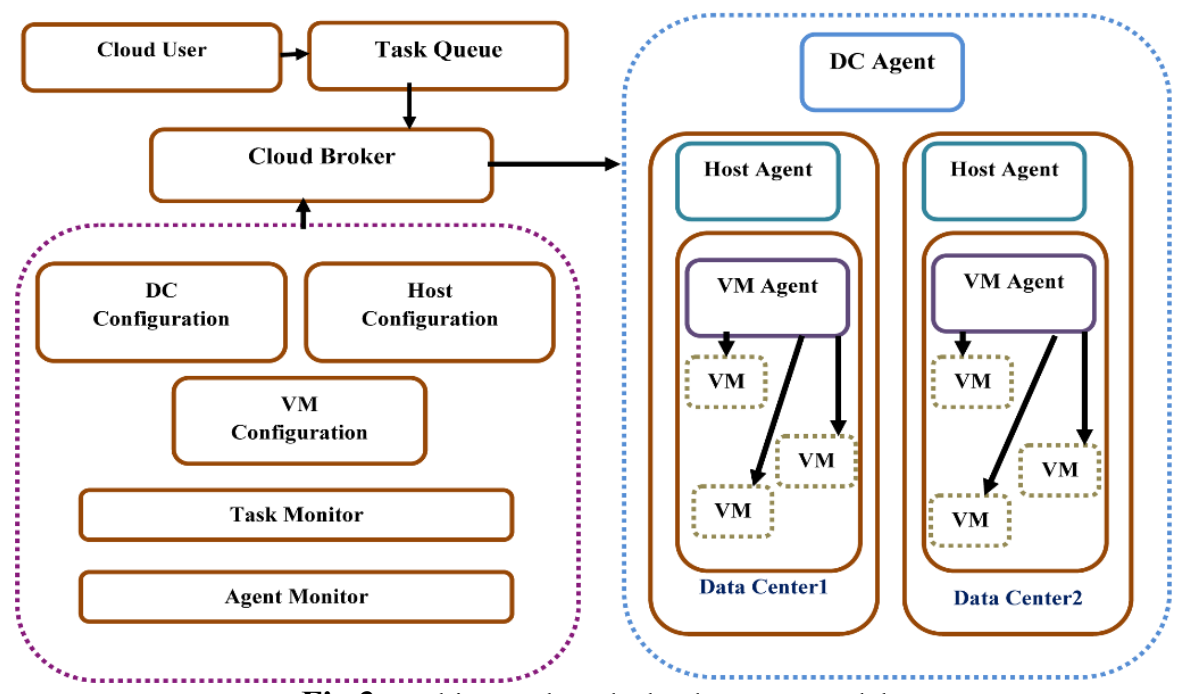

Fig.3. Multi agent based Cloud energy model

User module gets the task request that runs in the cloud. The cloud broker acts as a mediator between users and cloud. Configuration module specifies the various properties and attributes needed for minimizing the energy. Three kinds of configurations are considered namely Data Center (DC), Host and VM with corresponding monitoring and management process. Multi agent module performs respective selection based on the condition of the cloud resources by cloud agents. Cloud multi agent module identifies the unused or zero load resources and terminates the resources in order to calculate the energy level of other resources with minimum power. Cloud environment is organized as a DC that is maintained in various regions. DC agents collect the energy of various DCs that rely on the cloud environment. The host agent collects the energy level from various hosts in the data center. Virtual Machine (VM) agent receives the energy of various VM belongs to the specific host. The main objective of the proposed algorithm is to minimize the energy consumption of the cloud resources such as DC, Host and VM with high performance.

\section{VM Based Energy Analysis}

Distributed VM consolidation method with dynamic property finds the feasible solution by scaling the resources based on the energy constraints. The Existing static method maintains the centralized approach that increases the problem to find the optimal solution. 
This distributed approach supports in two ways namely VM management with predefined load limit and optimal VM placement. The integration of more than one agent uses the learning process with optimal energy saving and high efficiency in the cloud [11]. Multiple agents with intelligent capability are used over a single cloud environment to perform the learning process. This method chooses the next agent in case of failure in an existing agent using Q Learning approach. This method is also suitable for static type and dynamic type VM environments [12]. Advanced RL consolidation based agent minimizes the VM distribution over the cloud through efficient policy for management of VM. Dynamic environment faces problems due to the learning of optimal knowledge and uncertainty conditions [13]. The dynamic RL method reduces the active VM based on the energy level with policy based on the user requirement. This method uses the workload of the VM and its load history for making the VM idle state or termination state. It collects the log history dynamically because of the changes made in the cloud [14]. It fixes the threshold for the energy by collecting the VM list. Initially the VMA (VM Agent) issues the notification to the VMs for receiving the properties of the VM and corresponding level. Every VM in the VMA compares with the threshold by identifying the load of the VM. If it is zero and still it is in running state then remove that VM from the VM list and update the VMA. If the VM holds the minimum load then the capacity then perform the migration process for executing the task. Table 1 represents the deviation of the workload after allocation task.

\section{Pseudocode 1 VM Agent Energy Management ()}

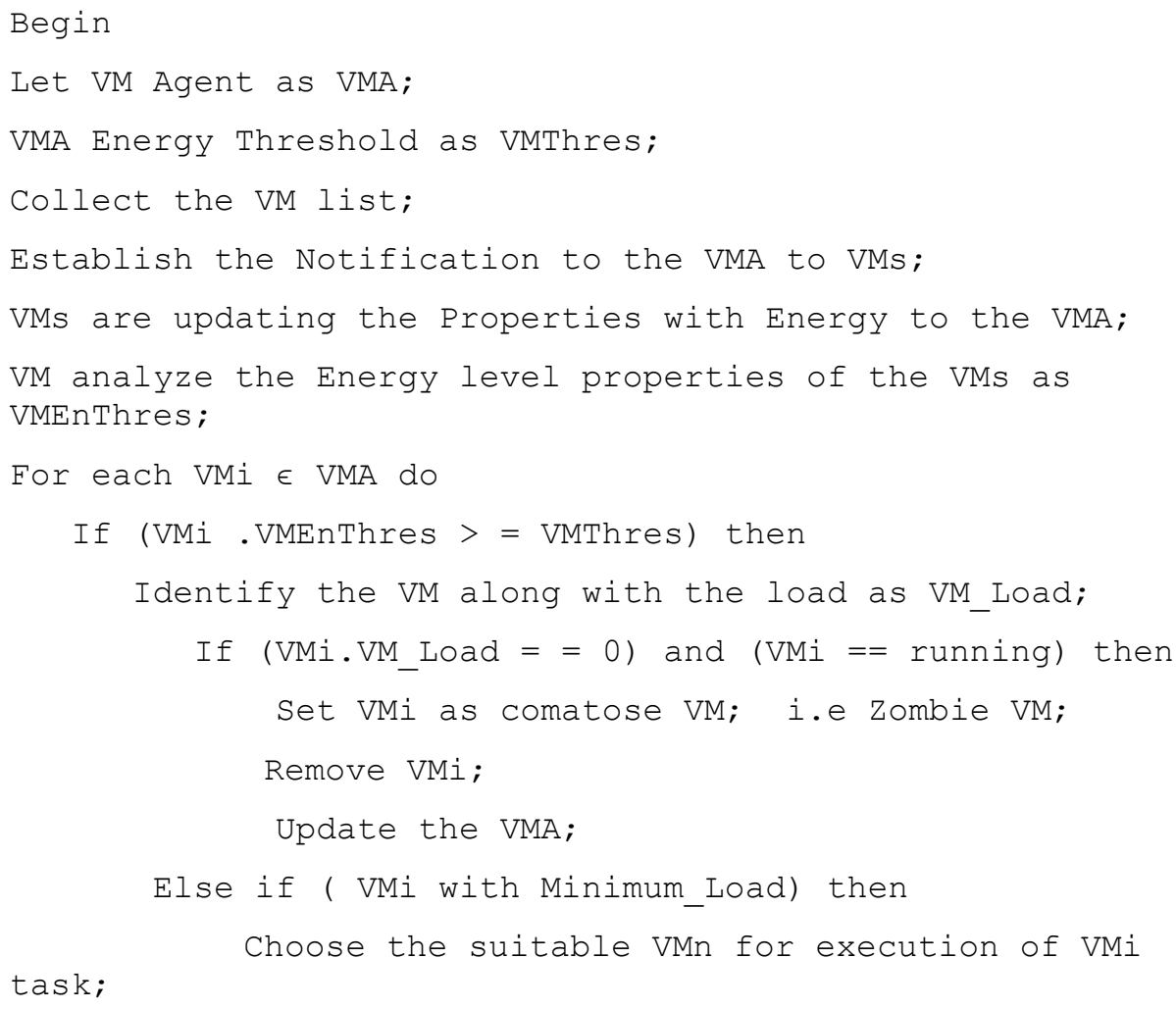




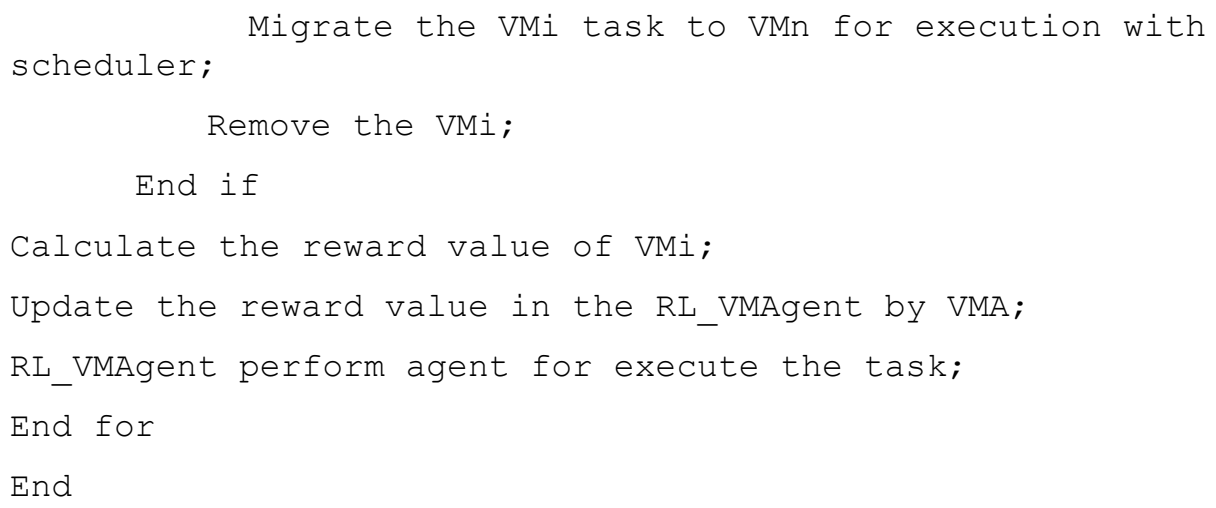

Table 1. Idle workload ratio analysis

\begin{tabular}{|c|c|c|c|c|c|}
\hline $\begin{array}{l}\text { Host } \\
\text { Name }\end{array}$ & $\begin{array}{l}\text { VM } \\
\text { Name }\end{array}$ & $\begin{array}{l}\text { Total } \\
\text { MIPS }\end{array}$ & $\begin{array}{l}\text { Allocated } \\
\text { Work } \\
\text { Load } \\
\text { MIPS }\end{array}$ & $\begin{array}{l}\text { Allocated } \\
\text { Work } \\
\text { Load } \\
\text { Ratio \% } \\
\end{array}$ & $\begin{array}{l}\text { Deviation } \\
\text { Ratio } \\
\text { Work } \\
\text { Load \% }\end{array}$ \\
\hline \multirow[t]{3}{*}{ Host 0} & $\begin{array}{l}\text { VM } \\
26\end{array}$ & 1000 & 545.09 & 54.51 & 45.49 \\
\hline & $\begin{array}{l}\text { VM } \\
27\end{array}$ & 1000 & 714.64 & 71.46 & 28.54 \\
\hline & $\begin{array}{c}\text { VM } \\
39\end{array}$ & 500 & 87.3 & 17.46 & 82.54 \\
\hline \multirow[t]{2}{*}{ Host 1} & VM 0 & 2500 & 601.34 & 24.05 & 75.95 \\
\hline & VM 1 & 2500 & 1025.2 & 41.01 & 58.99 \\
\hline \multirow[t]{3}{*}{ Host 2} & $\begin{array}{l}\text { VM } \\
28\end{array}$ & 1000 & 562 & 56.24 & 43.76 \\
\hline & $\begin{array}{l}\text { VM } \\
29\end{array}$ & 1000 & 731 & 73.19 & 26.81 \\
\hline & $\begin{array}{c}\mathrm{VM} \\
40\end{array}$ & 500 & 11.16 & 2.23 & 97.77 \\
\hline \multirow[t]{2}{*}{ Host 3} & VM 2 & 2500 & 2253 & 90.14 & 9.86 \\
\hline & VM 3 & 2500 & 177.48 & 7.1 & 92.9 \\
\hline \multirow[t]{3}{*}{ Host 4} & $\begin{array}{l}\text { VM } \\
30\end{array}$ & 1000 & 223.27 & 22.33 & 77.67 \\
\hline & $\begin{array}{l}\text { VM } \\
31\end{array}$ & 1000 & 393.81 & 39.28 & 60.72 \\
\hline & $\begin{array}{l}\text { VM } \\
41\end{array}$ & 500 & 95.93 & 19.19 & 80.81 \\
\hline
\end{tabular}

Our analysis of $50 \mathrm{VMs}$ is spinned out by the hypervisor. Each VM executes its own task based on the user requirement. The VMs are run under the hosts which decides the processing of the task. The actual energy threshold is specified in order to protect the resources from excess energy consumption. The energy consumption is assessed by using the metrics called $\mathrm{W}^{*}$ Sec. The energy is measured in watts with seconds. Idle VM list is identified from the host list and removes it for minimizing the energy. The total number of idle VM is 24 , so $\sim 50 \%$ of the energy is saved from the total energy. 


\section{Host Based Energy Analysis}

Host maintains the VM by considering the virtual private network with related components leads to congestion problems due to internal traffic. The private networks are divided into subnets either public or private for exposing the services run under the host. The host performs the offloading process by selecting the resource that consumes high power. RL based technique used to make the host respond to real time problem areas with less power consumption [15]. Cloud computing model uses various shared resources including host faces the issues during the consolidation process. It maintains the heterogeneous resources with respective environment needs Quality aware task scheduling to the corresponding VM for execution. RL algorithm analyses various algorithms for performing task-scheduling processes [16]. Power management in the cloud uses the migration process and consolidation of the resources based on the need of the consumer. The resources are going to be selected and terminated due to the dynamic workload condition of the cloud [17]. Host maintains various VMs along with the supporting networks which consume the energy. This is collected by the HA (Host Agent) for identifying the zombie hosts with zero load. This host is still in active state then removes it from the host list. Host consumes the energy based utilization parameter calculated with allocation of VM to the task. The idle hosts are identified from the data center and terminate it, because these hosts also consume the energy. The total energy consumption by the host without idle VM is 1890000.00 Watts/sec which is minimum than actual consumption.Distributed VM consolidation

\section{Data Center Energy Analysis}

The pricing model of the cloud energy management is related to the factors such as constraint in interaction, energy balance and objective function. Storage based energy management is done by integrating different storage with power consideration for improving the profit [18]. EM (Energy Management) systems are implemented by performing the operation like optimization, control and monitoring of energy. The cost of Industrial EM is high when compared to other EMs because of complex connectivity and interoperation. Native platform is used for addressing the issues of EM with minimum time [19]. Pattern based resource provisioning is done based on the scaling in a dynamic environment. Two levels of objectives are carried out in the cloud energy management process namely service level and application level. Service level agreement has been established for reducing overall energy with high efficiency [20]. Datacenter based power management is done using the suitable DC agent. The data centers are maintained in the same region or different regions, so monitoring related to energy consumption is a tedious process. The proposed model collects and updates the properties of various DC with proper threshold. If the status is active for zero workload then removes that DC and updates the DC list.

Table 2.Performance parameter analysis of the data centers

\begin{tabular}{|c|c|c|}
\hline \multirow{4}{*}{ Data Center 1 } & Number of hosts & 50 \\
\cline { 2 - 3 } & Number of VMs & 50 \\
\cline { 2 - 3 } & Energy consumption & $150.68 \mathrm{kWh}$ \\
\cline { 2 - 3 } & Number of host shutdowns & 29 \\
\hline
\end{tabular}




\begin{tabular}{|c|c|c|}
\hline \multirow{4}{*}{ Data Center 2} & Number of hosts & 45 \\
\hline & Number of VMs & 45 \\
\hline & Energy consumption & $143.57 \mathrm{kWh}$ \\
\hline & Number of host shutdowns & 27 \\
\hline \multirow{4}{*}{ Data Center 3} & Number of hosts & 40 \\
\hline & Number of VMs & 40 \\
\hline & Energy consumption & $139.51 \mathrm{kWh}$ \\
\hline & Number of host shutdowns & 30 \\
\hline \multirow{4}{*}{ Data Center 4} & Number of hosts & 35 \\
\hline & Number of VMs & 35 \\
\hline & Energy consumption & $133.73 \mathrm{kWh}$ \\
\hline & Number of host shutdowns & 28 \\
\hline \multirow{4}{*}{ Data Center 5} & Number of hosts & 30 \\
\hline & Number of VMs & 30 \\
\hline & Energy consumption & $128.58 \mathrm{kWh}$ \\
\hline & Number of host shutdowns & 26 \\
\hline \multirow{4}{*}{ Data Center 6} & Number of hosts & 25 \\
\hline & Number of VMs & 25 \\
\hline & Energy consumption & $126.62 \mathrm{kWh}$ \\
\hline & Number of host shutdowns & 21 \\
\hline \multirow{4}{*}{ Data Center 7} & Number of hosts & 20 \\
\hline & Number of VMs & 20 \\
\hline & Energy consumption & $123.55 \mathrm{kWh}$ \\
\hline & Number of host shutdowns & 18 \\
\hline
\end{tabular}

Data centers have various hosts, which are used to execute the task in various VMs. The analyses of different parameters are shown in Table 2. It includes the host count, VM count, energy and host shutdown count. The current analysis provides better energy saving when compared to actual consumption with the consideration of idle data centers.

\section{Result and Discussion}

Greencloud model provides the support of knowing the energy consumption of the datacenter. It also provides the specification such as allocation and scheduling of resources in an optimized manner. DVFS (Dynamic Voltage with Frequency Scaling) model reduces the energy of the data center in an autonomous manner. This method saves $41.62 \%$ of energy with the reduction of power and VM migration is $19.55 \%$ and $85.81 \%$ respectively [21] [24]. DVFS Aware model balances the issues of energy and degradation of performance in the cloud. It also focuses on the task workload related to the cloud resource capacity with high Quality of Services. This model saves the energy consumption in $39.14 \%$ under dynamic situations [22]. Scientific problems are solved by using the cloud because of heavy workload. If the workload is increased then cloud resources also increase in parallel manner. The EnReal model solved this problem by using energy aware scheduling and allocation of resources with minimum energy consumption. It consumes $24.45 \%$ energy for 50 scientific workloads when 
compared to BFD-M and Greedy methods [23]. The proposed multi agent algorithm follows maximum support of SLA when compared to existing methods. The Inter Quartile algorithm follows the minimum SLA rules, so it will be improved in some level. Inter Quartile and local regression robust method suffers performance issues due to the minimum SLA support which is shown in Fig.4. Non power aware methods consume more energy because they never consider energy parameters for analysis which means that all hosts in the data center take equal energy. Fig.5. shows that the proposed method consumes minimum energy due to the multi layered idle resource shutdown in the cloud.

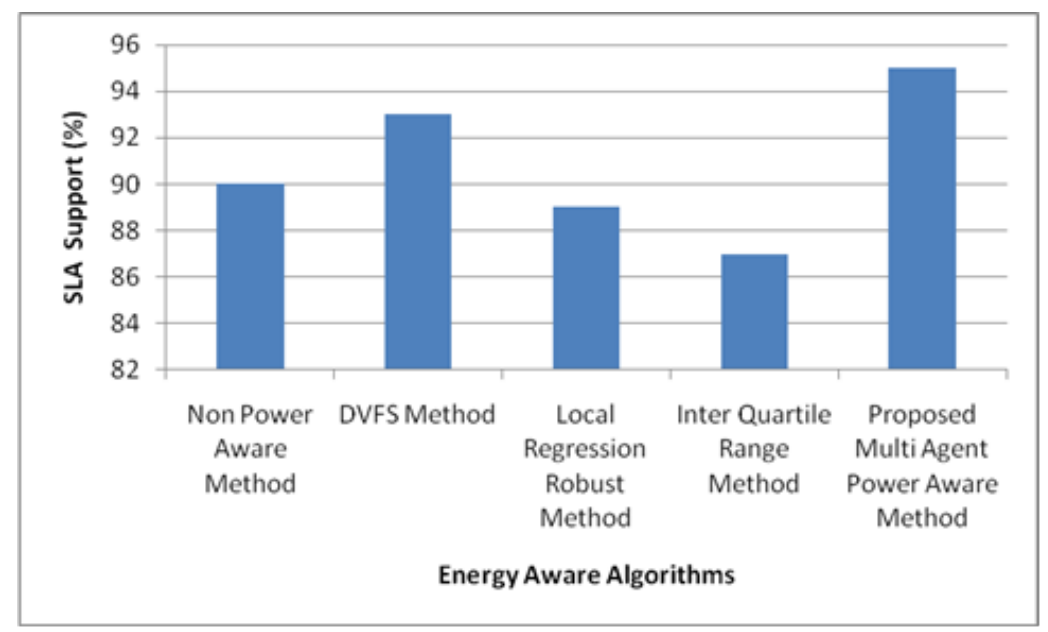

Fig.4.Analysis of SLA support

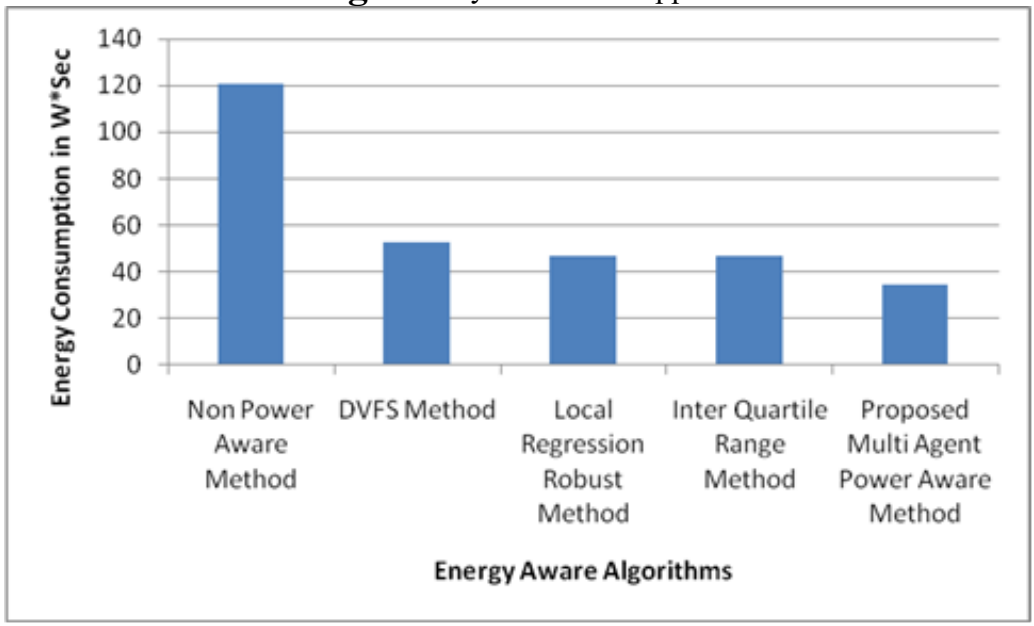

Fig.5.Analysis of Energy Consumption

\section{Conclusion and Future Work}


Cloud computing consumes energy because various kinds of resources are interacting to offer the service to the user. The resources lead to the performance issue, so it will be minimized in some level, consuming the excess energy. Various levels of resources are maintained by the cloud namely data center level, VM level and host level. The proposed model uses a multi agent based approach by handling the energy consumption at multiple levels. Efficient energy aware model has been implemented by eliminating the idle resource in all levels. VM based analysis is done with the help of a VM agent which identifies the zombie VMs and terminates from the VM list. Host agent removes the idle host and migrates less workload host to other hosts. DC agent has been implemented with minimized energy because it depends on the host as well as VM. Various algorithms are implemented in order to achieve better performance. The proposed model achieves minimized energy consumption when compared to existing models with high efficiency. In future this model can be extended to the RL based approach for various cloud environment with different analysis.

\section{References}

[1] Yashi Goyal, Meenakshi S. Arya,SunilNagpal,"Energy efficient hybrid policy in green cloud computing ", International Conference on Green Computing and Internet of Things (ICGCIoT), IEEE, 2015, Pp. 1065-1069.

[2] Takumi Sakamoto, Hiroshi Yamada, HikaruHorie, Kenji Kono,"Energy-Price-Driven Request Dispatching for Cloud Data Centers", IEEE Fifth International Conference on Cloud Computing, IEEE, 2012, Pp.974-976.

[3] Kumari ParulJasuja, Khushdeep Kaur, "Hybrid soft computing approach for energy efficiency in cloud computing", International Conference on Communication and Electronics Systems (ICCES), IEEE, 2016, Pp. 1-4.

[4] Lide Duan, Dongyuan Zhan, Justin Hohnerlein, "Optimizing Cloud Data Center Energy Efficiency via Dynamic Prediction of CPU Idle Intervals", IEEE 8th International Conference on Cloud Computing, IEEE, 2015, Pp. 985-988.

[5] AwatifRagmani ; Amina El Omri ; NoreddineAbghour ; Khalid Moussaid ; Mohammed rida,"An intelligent scheduling algorithm for energy efficiency in cloud environment based on artificial bee colony",3rd International Conference of Cloud Computing Technologies and Applications (CloudTech), IEEE, 2017, Pp.1-8.

[6] Subhra Priyadarshini Biswal, Satya Prakash Sahoo,"Fuzzy Logic Based Cost and Energy Efficient Load Balancing in Cloud Computing Environment",Second International Conference on Intelligent Computing and Control Systems (ICICCS), IEEE, 2018, Pp.158-163.

[7] Yongqiang Zhang, Jianbo He, SongtaoGuo,"Energy-Efficient Dynamic Task Offloading for Energy Harvesting Mobile Cloud Computing", IEEE International Conference on Networking, Architecture and Storage (NAS), 2018, IEEE, Pp.1-4.

[8] Tianchu Zhao, Sheng Zhou, Linqi Song, Zhiyuan Jiang, Xueying Guo, Zhisheng,"Energyoptimal and delay-bounded computation offloading in mobile edge computing with heterogeneous clouds",China Communications, Vol.17(5) 2020, Pp. 191-210.

[9] XiaominZhu,Laurence T. Yang, Huangke Chen, Ji Wang, Shu Yi, XiaochengLiu,"Real-Time Tasks Oriented Energy-Aware Scheduling in Virtualized Clouds", IEEE Transactions on Cloud Computing,Vol.2 (2), 2014, Pp.168 - 180.

[10] Dongyan Deng, Kejing He, Yanhua Chen,"4th International Conference on Cloud Computing and Intelligence Systems (CCIS)", IEEE, 2016, Pp. 366-370.

[11] SeyedSaeidMasoumzadeh, Helmut Hlavacs,"A Cooperative Multi Agent Learning Approach to Manage Physical Host Nodes for Dynamic Consolidation of Virtual Machines", IEEE Fourth Symposium on Network Cloud Computing and Applications (NCCA),IEEE, 2015, Pp.44-50.

[12] William Sause,"Coordinated Reinforcement Learning Agents in a Multi-agent Virtual Environment",12th International Conference on Machine Learning and Applications,IEEE, 2013.Pp. 227-230. 
[13] Rachael Shaw, Enda Howley, Enda Barrett,"An advanced reinforcement learning approach for energy-aware virtual machine consolidation in cloud data centers", 12th International Conference for Internet Technology and Secured Transactions (ICITST), IEEE, 2017, Pp. 6166.

[14] FahimehFarahnakian, PasiLiljeberg, JuhaPlosila,"Energy-Efficient Virtual Machines Consolidation in Cloud Data Centers Using Reinforcement Learning",22nd Euromicro International Conference on Parallel, Distributed, and Network-Based Processing, 2014, Pp. 500-507.

[15] Yunzhao Li, Feng Qi, Zhili Wang, Xiuming Yu, SujieShao,"Distributed Edge Computing Offloading Algorithm Based on Deep Reinforcement Learning" , IEEE Access,Vol. 8,IEEE, 2020, Pp. $85204-85215$.

[16] Lan Wang, Erol Gelenbe,"Adaptive Dispatching of Tasks in the Cloud", IEEE Transactions on Cloud Computing, Vol.6(1), IEEE, 2018, Pp.33 - 45.

[17] Shuai Wang, Xiaoqing Zhou, Mingsheng Shang, XiaoyuShi,"Coordinated Power and Performance-Efficient Virtual Machines Scheduling in the Cloud",10th International Conference on Communications, Circuits and Systems (ICCCAS),IEEE, 2018, Pp.489-494.

[18] Lulu Jiang, Renjun Zhou, Jiangsheng Zhu, Jian Xu, YanyanXu,"Electricity Charge Saved for Industrial and Commercial Utilizing Cloud Energy Storage Services",3rd Conference on Energy Internet and Energy System Integration (EI2),IEEE, 2019, Pp. 1113-1116.

[19] Hugo Sequeira, Paulo Carreira, Thomas Goldschmidt, Philipp Vorst, "Energy Cloud: RealTime Cloud-Native Energy Management System to Monitor and Analyze Energy Consumption in Multiple Industrial Sites", IEEE/ACM 7th International Conference on Utility and Cloud Computing, IEEE, 2014, Pp. 524-534.

[20] Li Xu, Guozhen Tan, Xia Zhang, JingangZhou,"Energy aware cloud application management in private cloud data center",International Conference on Cloud and Service Computing, IEEE, 2020, Pp. 274-279.

[21] Patricia Arroba, José M. Moya, José L. Ayala, Rajkumar Buyya, "Dynamic Voltage and Frequency Scaling-aware dynamic consolidation of virtual machines for energy efficient cloud data centers", Concurrency Computation: Practs Experience, Wiley, 2016, Pp. 1-13.

[22] Patricia Arroba, José M. Moya ,José L. Ayala, Rajkumar Buyya, DVFS-Aware Consolidation for Energy-Efficient Clouds , International Conference on Parallel Architecture and Compilation, 2015, IEEE, Pp.494-495.

[23] Xiaolong Xu, Wanchun Dou, Xuyun Zhang, Jinjun Chen,"EnReal: An Energy-Aware Resource Allocation Method for Scientific Workflow Executions in Cloud Environment", IEEE Transactions on Cloud Computing, Vol.4(2), 2016,Pp. 166 - 179

[24] Anton Beloglazov, and Rajkumar Buyya, "Optimal Online Deterministic Algorithms and Adaptive Heuristics for Energy and Performance Efficient Dynamic Consolidation of Virtual Machines in Cloud Data Centers", Concurrency and Computation: Practice and Experience (CCPE), Volume 24, Issue 13, Pages: 1397-1420, John Wiley \& Sons, Ltd, New York, USA, 2012

[25] N. H. Motlagh, M. Bagaa and T. Taleb, "Energy and Delay Aware Task Assignment Mechanism for UAV-Based IoT Platform", IEEE Internet of Things Journal, vol. 6, no. 4, pp. 6523-6536, Aug. 2019.

[26] O. Yilmaz, E. Yakici and M. Karatas, "A UAV location and routing problem with spatiotemporal synchronization constraints solved by ant colony optimization", Journal of Heuristics, vol. 25 , pp. $673-701,2019$. 